\title{
Large-scale Cryogenic Gravitational wave Telescope
}

\section{LCGT Collaboration}

Kazuaki Kuroda $^{1}$, Masatake Ohashi, Shinji Miyoki, Daisuke Tatsumi, Shuichi

Sato, Hideki Ishizuka

ICRR, The University of Tokyo, 3-2-1, Midoricho, Tanashi, Tokyo 188-8502

Masa-Katsu Fujimoto, Seiji Kawamura, Ryutaro Takahashi, Toshitaka Yamazaki, Koji Arai, Mitsuhiro Fukushima, Koichi Waseda, Souichi Telada, Akitoshi Ueda

NAO, 2-21-1, Ohsawa, Mitaka, Tokyo 181-8588

Takakazu Shintomi, Akira Yamamoto, Toshikazu Suzuki, Yoshio Saito,

Tomiyoshi Haruyama, Nobuaki Sato

KEK, 1-1, Oho, Tsukuba, Ibaraki 305-0801

Kimio Tsubono, Keita Kawabe, Masaki Ando

Department of Physics, The University of Tokyo, 7-3-1, Hongo, Bunkyo, Tokyo 113-0033

Ken-ichi Ueda, Hitoki Yoneda, Mitsuru Musha

Institute for Laser Science, The University of Electro-Communications, 1-5-1, Choufugaoka, Choufu, Tokyo 182-8585

Norikatsu Mio, Shigenori Moriwaki

Department of Material Science, The University of Tokyo, 7-3-1, Hongo, Bunkyo, Tokyo 113-8656

Akito Araya

ERI, The University of Tokyo, 1-1-1, Yayoi, Bunkyo, Tokyo 113-0032

Nobuyuki Kanda

Miyagi University of Education, Aoba, AzaAramaki, Aoba-ku, Sendai

Mike E. Tobar

Department of Physics, The University of Western Australia, Nedlands 6009, Australia

\begin{abstract}
We present here the Large-scale Cryogenic Gravitational wave Telescope (LCGT) project which is aimed to improve the sensitivity of the existing gravitational wave projects by ten times. LCGT is the project constructing the $\mathrm{km}$-scale gravitational wave detector in Japan succeeding the TAMA project, which adopts cryogenic mirrors with a higher power laser. We are planing to build it in an underground site in Kamioka mine. If its target sensitivity is attained, we will be able to catch a few events per month.
\end{abstract}

\footnotetext{
${ }^{1}$ E-mail: kuroda@icrr.u-tokyo.ac.jp
} 


\section{Introduction}

In the beginning of the next century, there will be three interferometric gravitational wave detectors of km-scale baseline and two of intermediate size. LIGO (USA collaboration for gravitational wave detection) will have two interferometers of $4 \mathrm{~km}$ baseline: one in Hanford, Washington and the other in Livingston, Louisiana [1]. VIRGO, a French and Italian collaboration, will have a three km-sized one in Pisa, Italy [2]. GEO, a German and English collaboration, will operate $600 \mathrm{~m}$ baseline interferometer in Hanover in Germany [3] and TAMA will have a $300 \mathrm{~m}$ interferometer in Mitaka, Japan [4]. The main target of these is an event involving the coalescence of a neutron-star binary; its wave form is known in a relatively precise way, which is used to make a matched filter to enhance the detectable signal-to-noise ratio $(\mathrm{S} / \mathrm{N})$. At the initial phase of the projects, these $\mathrm{km}$-scale interferometers are expected to have a possibility to catch events occurring in Virgo cluster, 20 Mpc away from the Earth. A statistical study shows that the birth rate of which coalescence is $10^{-6}$ a year in a galaxy as big as our Galaxy [5]. This means that we should wait to catch another event for two hundred and fifty years after the first detection of a gravitational wave with the most sensitive detector in the world, since there are four thousand galaxies of our Galaxy size in the Virgo cluster. If we are not lucky to catch the first event a few years after construction, we will need to wait for a time span of a century on the average.

Therefore, it is clear to everyone much more sensitive detectors must be developed to see more remote galaxies. If we increase the sensitivity by ten times, the average interval will become three months, since the coverage space is linearly proportional to its sensitivity, considering one galaxy per one cubic Mpc. A ten-times improvement may be a dream at present. Because those existing plans adopt the most advanced technique to attain the best sensitivity in their designing stages, it is not easy to design more sensitive detectors with the existing techniques.

We present here the Large-scale Cryogenic Gravitational wave Telescope (LCGT) project to improve the sensitivity by ten times. Since thermal noise is one of important limiting issues, this detector adopts a cryogenic technique with a higher power laser. After two years of development of the cryogenic system, we have reached a stage where we can begin to start constructing the cryogenic interferometric detector. This detector is the km-scale gravitational wave detector project in Japan, succeeding the TAMA project, and we are planing to build it in an underground site near Super KAMIOKANDE in Kamioka mine. Figure 1 shows the detection range of LCGT compared with that of other projects.

In this document, we describe the significance of the detection of gravitational waves, the current status of the TAMA project and the design of LCGT with R\&D of important technologies, including the cryogenic mirror. Finally, we propose an organization to realize the LCGT along with a cost estimation. 


\section{View ranges of Gravitational wave detectors in the world}

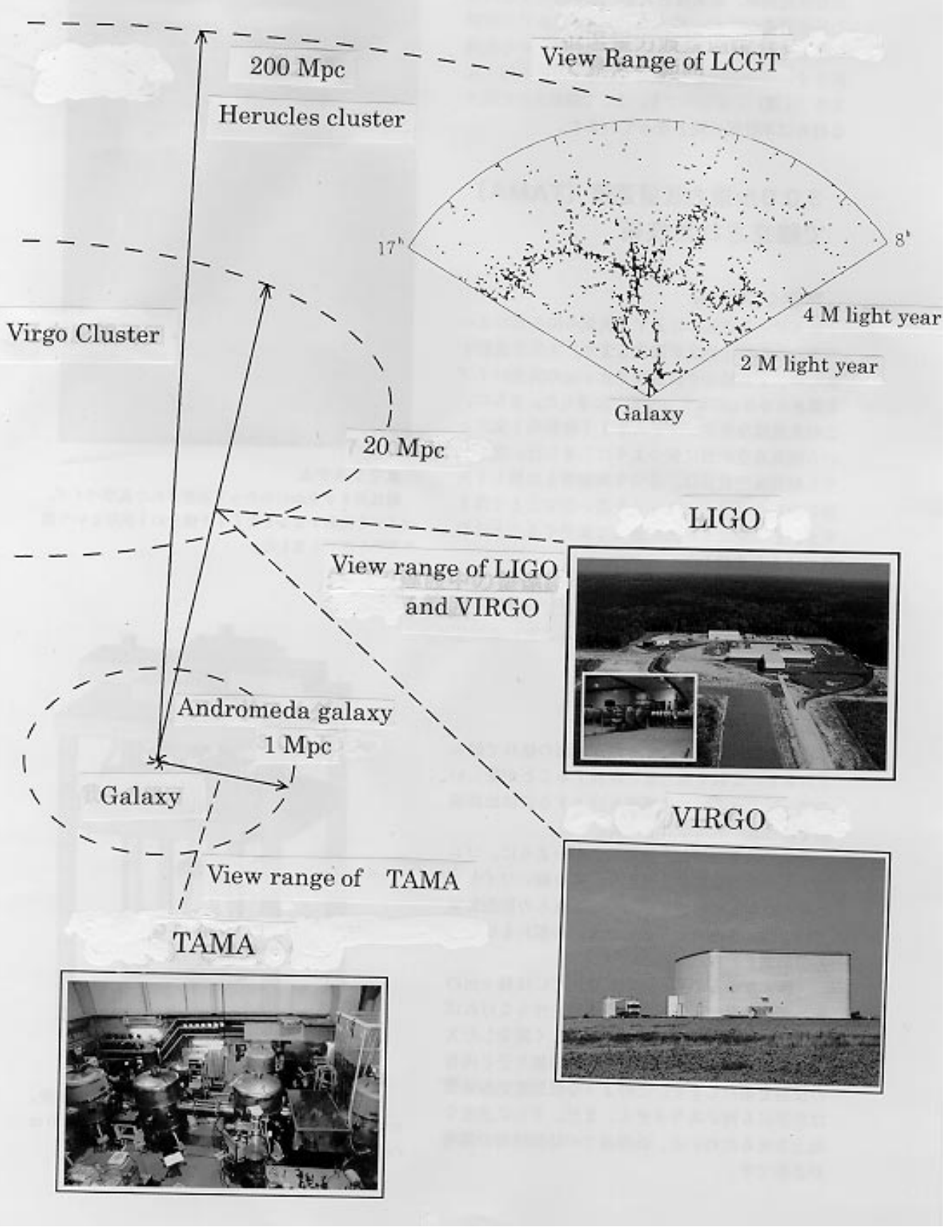

Figure 1: View ranges of gravitational wave detectors in the world. LCGT can catch events occurring $200 \mathrm{Mpc}$ away from the Sun in $\mathrm{S} / \mathrm{N}=10$. This is more sensitive by about 10 times than that of LIGO or VIRGO. 


\section{Physical Meaning of Gravitational Wave De- tection}

A gravitational wave is a physical entity in spacetime derived from Einstein's theory of general relativity. Its existence was indirectly proved by the observation of PSR1913+16 by Taylor and Hulse [6], who won the Nobel prize in 1993. Nobody has succeeded in detecting a gravitational wave directly. The theory of general relativity has been tested so far in our solar system, where the effect of gravitation is very small. These tests are the periphelion shift of Mercury, the bending of light rays passing near by the hem of the Sun and the Shapiro delay of a radar echo. The former phenomenon was clearly observed in the binary system of PSR1913+16, because the gravitation there is greater than that of our solar system by more than three orders of magnitude.

The detection of a gravitational wave is a direct test of general relativity in a strong gravitational field. Since the field equation of general relativity is highly nonlinear, an analytical calculation needs some linearization that may introduce an uncertainty in the final result. Especially, only one unappropriate approximation may cause a serious error in such an extremely strong field. In place of an analytical calculation, a numerical method is applied to such highly nonlinear dynamics as a binary neutron star coalescence. However, only a few cycles at the beginning stage of coalescence have been calculated, and it is hard to see the emission of gravitational waves due to a lack of computation power. Therefore, the direct detection of gravitational wave will present not only a proof of the theory of general relativity, but also an unknown aspect of the coalescence of binary systems.

Moreover, the technique to catch gravitational wave events can be applied to observe the Universe. This is the last eye of mankind to inspect the Universe. A global network of three detectors can basically determine the position of a coalescence and their Keplerian parameters, including masses. Since the directional sensitivity may degrade the accuracy, we need at least four detectors on the Earth. And if the quality of the signal is good enough to determine these parameters, the first impact to the field of astronomy is the accuracy of the distance, which may be used to re-determine the Hubble constant with an accuracy never reached so far.

The general relativity of Einstein is not the sole theory of gravitation. The Brans- Dicke theory is still alive, and many other theories have not been completely rejected by experiments [7]. If we consider this Brans-Dicke theory, gravitational waves would have three degrees of freedom in place of two in the case of general relativity. Four sensitive detectors would settle whether this is correct or not. Such a test may disclose the profound nature of gravitation.

We give priority to the detection of an event of neutron star binary coalescence in this LCGT project. However, there are many other sources of gravitational waves. They are supernova explosions, continuous waves from pulsars, blackhole binary coalescence, falling object into blackholes, cosmic strings, domain walls in early Universe, background radiation, and so on. Among these, supernova explosions and continuous waves from pulsars are only within the scope of the Earth-based interferometric detectors that are sensitive to events having frequencies from $100 \mathrm{~Hz}$ to $1000 \mathrm{~Hz}$. The theoretical waveform has not yet been clearly calculated for the supernova process. If we know the waveform 
beforehand, the probability to catch events increases extensively because we have many possible sources, even near to our Galaxy. As for a continuous wave, the magnitude is estimated to be fairly small, and we have not found any pulsars with a high spin-down rate that is a possible strong source. On the other hand, if we catch one of these events, it could contribute to disclose the physics of pulsar dynamics. Even though the other sources may not be detected by Earthbased detectors, it might be possible by using detectors in space, because these sources have a characteristic frequency of less than $1 \mathrm{~Hz}$ and Newtonian gravitational noise limits the sensitivity in low-frequency region of less than $10 \mathrm{~Hz}$ on the Earth. A space project, called LISA, will have a prototype satellite in space early next century. However, we believe that we must finish the Earth-based detectors first and let them run for monitoring gravitational waves on the Earth.

\section{Detection of gravitational wave}

When a gravitational wave passes through space where two free masses are placed, a distance between them changes, and if the two masses are firmly connected together, a force is produced between them. If the gravitational wave was produced by an event in the VIRGO cluster and it was the coalescence of a binary neutron star, the change in the distance on the Earth is $\sim 10^{-21} \mathrm{~m}$ for one-meter displacement. In the typical baseline length of $3 \mathrm{~km}$, it becomes $\sim 3 \times 10^{-18} \mathrm{~m}$. This is extremely small, but can be detected.

Since the displacement change due to a gravitational wave is extremely small, its detection is mainly contaminated by mechanical vibrations, regardless of the detection scheme. Generally speaking, the detection scheme consists of transforming the displacement to an electric signal. In this detection, quantum shot noise, for example, photon shot noise is added to the above mechanical noise. At a low frequency of less than $30 \mathrm{~Hz}$ seismic noise cannot be completely reduced through anti-vibration systems, which becomes dominant in the sensitivity spectrum of the detector. In the mid band from $30 \mathrm{~Hz}$ to $300 \mathrm{~Hz}$, thermal vibration of the mirror, or the suspension system, is dominant. In the region higher than $300 \mathrm{~Hz}$ photon shot noise dominantly appears. To each frequency band we have developed the most suitable technique to reach each level of the ultimate noise.

Nobody doubts that the target sensitivity is attained at the end of the construction of the present projects, even though technical obstacles may delay its schedule. Since the principle of new technology for LCGT has been confirmed to properly work [8], we can surely attain the final object of LCGT without more advanced special techniques that need new developments hereafter.

\section{Result of TAMA}

TAMA is a project to construct a $300 \mathrm{~m}$ baseline laser interferometer at Mitaka campus of National Astronomical Observatory and to conduct observations. We regard the TAMA interferometer being as one step to reach the final scale interferometer in the sense of technology and the construction budget. During the course of this construction, we have established the techniques necessary for a large-scale and higher sensitive interferometer. We are confident that the 
TAMA project will be successful and several techniques developed so far are promising, as reported below.

The optical configuration of TAMA is a Fabry-Perot-Michelson laser interferometer with a power-recycling scheme [4]. Although the designed sensitivity is $2 \times 10^{-22} / \sqrt{ } \mathrm{Hz}$ in the root power spectrum of the relative displacement, it is expected to achieve $6 \times 10^{-22} / \sqrt{ } \mathrm{Hz}$ later, considering precise estimation of the thermal noises of the main mirrors. With this sensitivity we can catch a coalescence signal from Andromeda by $\mathrm{S} / \mathrm{N}=3$. Even with a half year delay, the project is steadily proceeding and will give the first and the most sensitive noise spectrum this October (1999).

\subsection{Vacuum system}

In the design of the vacuum system, we considered how to apply this experience to a future large-scale system. In such a large vacuum system as a $\mathrm{km}$-scale one, the work of baking will not be an easy task. Therefore, we took a policy of a non-baking system, even for the intermediate scale of TAMA. Table 1 shows the attained levels of the vacuum vacant chambers and ducts that are labeled in Fig. 2. In machining of the vacuum system, we applied a surface treatment onto the surface of the vacuum ducts and chambers for an ultra-high vacuum. The ducts and almost all chambers were made of stainless steel and were polished by electro-chemical buffing [11]. Two of chambers were made of titanium for a test. One of two measurements with higher pressure came from surface contamination (NM 1) and the other from the low pumping speed (MC 2), which was improved when stacks were installed (in the right row). This vacuum system is fairly good for a non-baking system, and we believe that this technique is promising for the final $\mathrm{km}$-scale vacuum system. One important acquirement was that the longer time the ducts were stacked after machining, the worse the quality of gas releasing in vacuum became.

\subsection{Anti-vibration and suspension system}

The present anti-vibration system consists of stacks and a double-pendulum suspension; also, an X-isolator for low frequency will be installed at the end. The stack has three disk blocks of stainless steel with chloroprene rubber housed in a bellows sealing. Three stacks support an optical breadboard. The frame which suspends the double-pendulum suspension is set on this board. The mirror is suspended by two loops of tungsten wire from an aluminum block, which is also suspended by four wires through a vertical isolation spring. The final base is attached to the frame. The base has adjustments of three degrees of freedom $(\mathrm{x}, \mathrm{y}, \mathrm{z})$ and pitch. Figure 3 shows this suspension on the optical breadboard. The transfer function is shown in Fig. 4, where measurements were applied to each stage of vibration isolation and composed. Many minor improvements were applied to the suspension system during the development and construction. The long-term stability of the rubber stack was also confirmed to be applicable for an practical system. For a future improvement we plan to install an X-isolater. 
Table 1: Measured vacuum pressure of the TAMA vacuum system. The labels show each duct and chamber given in Fig. 2. When the duct was opened to chambers, steady state vacuum pressure was $2 \times 10^{-6} \mathrm{~Pa}$ without turbo molecular pumping. UNIT: $\mathrm{Pa}$

\begin{tabular}{|c|c|c|}
\hline & Empty & With Stack \\
\hline \multirow[t]{2}{*}{ MC1 } & $7 \times 10^{-6}$ & $1 \times 10^{-5}$ \\
\hline & $(1000 \mathrm{~L}$ TMP $+400 \mathrm{~L}$ TMP $)$ & $(1000 \mathrm{~L}$ TMP) \\
\hline \multirow[t]{2}{*}{$\mathrm{MC} 2$} & $3 \times 10^{-5}$ & $1 \times 10^{-5}$ \\
\hline & $(300 \mathrm{~L}$ TMP $)$ & $(1000 \mathrm{~L}$ TMP) \\
\hline \multirow[t]{2}{*}{ EM1 } & $3 \times 10^{-6}$ & $6 \times 10^{-6}$ \\
\hline & $(1000 \mathrm{~L} \mathrm{TMP}+400 \mathrm{~L} \mathrm{TMP}+800 \mathrm{~L} \mathrm{IP})$ & $(1000 \mathrm{~L} \mathrm{TMP}+400 \mathrm{~L} \mathrm{TMP}+800 \mathrm{~L} \mathrm{IP})$ \\
\hline \multirow[t]{2}{*}{ NM1 } & $2 \times 10^{-5}$ & $3 \times 10^{-5}$ \\
\hline & (1000L TMP) & (1000L TMP) \\
\hline \multirow[t]{2}{*}{ EM2 } & $6 \times 10^{-6}$ & $6 \times 10^{-6}$ \\
\hline & (1000L TMP) & (1000L TMP) \\
\hline \multirow[t]{2}{*}{ NM2 } & $9 \times 10^{-6}$ & $2 \times 10^{-5}$ \\
\hline & $(1000 \mathrm{~L}$ TMP) & $(1000 \mathrm{~L}$ TMP) \\
\hline \multirow[t]{2}{*}{ BS-RM } & $2 \times 10^{-5}$ & $2 \times 10^{-5}$ \\
\hline & $(1000 \mathrm{~L}$ TMP $+400 \mathrm{~L}$ TMP $)$ & $(1000 \mathrm{~L}$ TMP $+400 \mathrm{~L}$ TMP $)$ \\
\hline \multirow[t]{2}{*}{ W-DCT } & $2 \times 10^{-6}$ & \\
\hline & $(1000 \mathrm{~L} \mathrm{TMP} \times 2+800 \mathrm{~L} \mathrm{IP} \times 2)$ & \\
\hline \multirow[t]{2}{*}{$\mathrm{E}-\mathrm{DCT}$} & $4 \times 10^{-6}$ & \\
\hline & $(1000 \mathrm{~L} \mathrm{TMP} \times 2+800 \mathrm{~L} \mathrm{IP} \times 2)$ & \\
\hline \multirow[t]{2}{*}{ S-DCT } & $1 \times 10^{-6}$ & \\
\hline & $(1000 \mathrm{~L} \mathrm{TMP} \times 2+800 \mathrm{~L} \mathrm{IP} \times 2)$ & \\
\hline \multirow[t]{2}{*}{ N-DCT } & $2 \times 10^{-6}$ & \\
\hline & $(1000 \mathrm{~L} \mathrm{TMP} \times 2+800 \mathrm{~L} \mathrm{IP} \times 2)$ & \\
\hline
\end{tabular}




\subsection{Laser and Optical system}

The laser source was developed by Sony Corporation under a contract with TAMA. It is an injection-locked Nd:YAG laser with a wavelength of $1.06 \mu \mathrm{m}$. The master laser is a commercial NPRO with an output power of $700 \mathrm{~mW}$. This is injected to a ring cavity of the slave laser pumped by two $12 \mathrm{~W}$ fiber-coupled LDs, as shown in Fig. 5 [12]. The maximum output power was almost $10 \mathrm{~W}$. The measured amplitude fluctuation was fairly small above a frequency of a few hundred Hz. Since we used this laser system at a frequency of about $15 \mathrm{MHz}$, it satisfies the specification. A further reduction of noise has been attained by feedback control subsystems.

The basic optical system is shown in Fig. 6. The laser light is introduced into a ring-type mode cleaner where modes other than $\mathrm{TEM}_{00}$ are suppressed. The output of the mode cleaner is fed into the Michelson part of the interferometer through the recycling mirror. In the first experiment of recombination, this recycling mirror will not be installed. The laser beam is split by the beam splitter and injected to both of the main Fabry-Perot cavities. The designed finesse of these cavities is about 500 and the light power of the cavity is $500 \mathrm{~W}$ each without recycling and $9 \mathrm{~kW}$ with recycling. We apply the frontal modulation method to remove any optical modulator inside the main optical paths. The modulated laser light is passed through the mode-cleaner cavity by matching the resonance [13]. The orientations of main mirrors are automatically controlled to maintain good alignment by the wave front sensing technique [15]. This was turned to be a powerful tool to obtain the stable operation of this interferometer [14].

\subsection{Interferometer Experiment}

All optical pieces, except those parts for recycling, had been installed by the end of August, 1998, and the first interference test was successfully conducted in September, 1998. Each arm was independently locked and the error signal of one arm was fed back into the laser source $(700 \mathrm{~mW}$, NPRO) for frequency stabilization. After installing the mode-cleaner with the $10 \mathrm{~W}$ laser, the first operation of recombination scheme without recycling was done in March, 1999. By adding small changes, short-term stability was accomplished in May, 1999. Figure 7 shows the current noise spectrum curve in this operation. Although the ultimate sensitivity has not yet been attained, the minimum noise has reached to $10^{-16} \mathrm{~m} / \sqrt{ } \mathrm{Hz}$ in $500 \mathrm{~Hz}$. This is a promising result.

\subsection{For the final operation}

By September, 1999, the full detector without recycling will be tested with the help of the data-acquisition system for a time scale of one week. After a minor change or improvement, a recycling technique will be applied in November, 1999.

\section{Design Policy of LCGT}

The target sensitivity of LCGT is to catch binary neutron star coalescence events occurring at $200 \mathrm{Mpc}$ with $\mathrm{S} / \mathrm{N}=10$. This is more sensitive by ten times than 
Table 2: Main parameters of LCGT

$\begin{array}{rc}\text { Item } & \text { Parameter } \\ \text { Baseline Length } & 3 \mathrm{~km} \\ \text { Interferometer } & \text { Fabry-Perot-Michelson with power recycling } \\ \text { Optical Power } & \text { laser: } 100 \mathrm{~W} \text {, effective power: } 2500 \mathrm{~W} \\ \text { Recycling Gain } & 50 \\ \text { Mirror } & \text { optical loss: } 1 \text { ppm, diameter: } 30 \mathrm{~cm}, \\ & \text { mass: } 54 \mathrm{~kg}, \text { material: sapphire } \\ \text { Acoustic } Q & \text { mirror internal: } 10^{7}, \text { pendulum: } 10^{7} \\ \text { Vacuum } & \leq 10^{-6} \mathrm{~Pa}\end{array}$

that of the first LIGO and by two orders compared to that of TAMA at the most sensitive frequency. The noise spectrum at $100 \mathrm{~Hz}$ is nearly limited by the quantum limit set by the uncertainty principle of the motion of the mirror mass. This is attained by the three-kilometer baseline, cooling mirrors to the cryogenic temperature, high-power laser source of $100 \mathrm{~W}$, and higher recycling gain. The optical configuration succeeds that of TAMA, which is a Fabry- PerotMichelson interferometer with techniques of the frontal modulation, wave-front sensing, and power recycling.

According to the longer baseline of the main Fabry-Perot cavity, the inner diameter of the vacuum duct has to be enlarge by three-times that of TAMA and optical baffles must be installed to prevent scattered light from interfering with the main beam, which would possibly produce excess noise. An ultra-high vacuum is also needed, but we will not apply baking because we have developed a non-baking technique for TAMA with successful results. We have adopted sapphire for the mirror material and suspension fibers. Its internal mechanical loss, that determines the magnitude of thermal noise, is smaller than that of fused silica, and is especially much smaller in cryogenic temperature, which guarantees smaller thermal noise with reduced temperature. The heat conductivity of sapphire is also better and increasing more in cryogenic temperature; this fact eases the thermal lens effect. Since this characteristic of sapphire is promising, even in room temperature, there is an opinion that one does not need to cool it down to obtain the present target sensitivity, and only critical parts should be cooled, for example, the suspension wire, because the pendulum thermal fluctuation must be removed by reducing its temperature. However, thermal noise of the mirrors is one of the ultimate obstacles in making the sensitivity higher, and we have learned from the long development of cryogenic resonant antennae that if there is a temperature gradient, pulse-like acoustic noise is issued, which must be avoided. A high output power laser will be accomplished by amplifying the output of an injection-locked master-slave laser, which realized an output of $10 \mathrm{~W}$ for TAMA. Since the total loss of the interferometer system determines the recycling gain, we continue to develop high-quality optical coating and mirror-polishing improving the internal optical loss of optical parts.

Table 2 lists the main parameters of LCGT. Figure 8 represents the target sensitivity of LCGT along with those of other projects.

We describe here the designs of the main parts of LCGT (the vacuum system, the mirror suspension system and the high power laser system) in detail. 


\subsection{Vacuum System}

Since this LCGT is to be built in the ground of Kamioka mine, the system needs to be designed to meet this requirement. The environmental conditions underground include space limitations, high humidity and stable ambient temperature. From the point of maintenance cost, only chambers of the main mirrors are to be cooled with vacuum ducts remaining at room temperature. In this scheme there are two problems: one is heat radiation incoming to the cryogenic mirror and the other is the deposition of residual gas molecules onto the mirror, that degrades optical quality. Heat radiation can be estimated when the chamber design is fixed; a rough estimation shows that about a $10 \mathrm{~m}$ radiation shield is needed between the mirror chamber and the vacuum duct to suppress the effect of blackbody radiation incoming from the duct at room temperature. Molecule deposition onto the mirror was qualitatively confirmed by an experiment [16], where the contamination disappeared when the mirror temperature was raised to room temperature again. Although a radiation shield is effective to reduce mirror contamination by molecular flux, it is not enough to maintain a high optical quality for long observational times. Therefore, we have designed a molecule purge system that works at room temperature. The basic idea is to raise the temperature of the main mirrors for purging. We will install a pair of interferometers working alternatively for preventing the detector from stopping observations during a contamination purge of the main mirrors of each interferometer. This idea is included in the design of the vacuum system, as shown in Fig. 9.

Since the vacuum chambers are evacuated before cooling down to cryogenic temperature, mirror contamination must be avoided at the beginning of the evacuation. Although an ultra-high vacuum is attained under normal operation of the cryogenic temperature not by ultra-high-vacuum chamber, the inner surface and vacuum materials have to be for ultra high vacuum against this initial contamination. To the inner surface of the duct will be applied ElectroChemical-Buffing (ECB) and may be coated with TiN. Turbo-molecular pumps are to be used at the beginning stage of evacuation. Ion pumps are to be used under normal operation.

\subsection{Mirror-suspension System}

The design of the suspension system is based on the R\&D being conducted with KEK Cryogenic Science Center. Since the mirror is suspended by fine fibers, there is no way to cool it by these fibers, because heat transfer by radiation ceases to be effective at temperature as low as $20 \mathrm{~K}$ or less. A high vacuum also prevents heat convection. Therefore, heat conduction through the fibers is the only way to cool, as described in the section concerning R\&D in this document. A schematic diagram of the suspension is shown in Fig. 10, where only part of the end-mirror chamber is focused. The mirror is suspended by one or two loops of sapphire fibers connected to an alignment control platform that has a heat link to the liquid helium tank inside the vacuum, which is put just above the platform. We are studying two types of suspensions, which leaves ambiguity of the system at present. This platform is suspended with a G-10 (thermal insulator) rod connected through the center hole of the helium tank to an isolation table suspended by a double $\mathrm{X}$-isolator. Cryogenic helium gas is 
used to maintain low temperature by circulation in super-insulator layers. All of these cryogenic system and $\mathrm{X}$-isolator are put inside a high vacuum. The support of the $\mathrm{X}$-isolator is put on the bottom of the upper chamber, the base of which is suspended from the ceiling of the tunnel.

Since the acoustic $Q$ of the mirror is as high as $10^{8}$, a new actuation system is needed, because the traditional permanent magnet actuator degrades the $Q$ of the mirror due to mechanical loss arising from bonding onto the mirror. Such a possible actuator utilizes the Meissner effect, which is effective at cryogenic temperature, where a few spots of thin film for super-conductance are sputtered on the mirror.

\subsection{Optical Technique}

Basically, the optical configuration is the same as that of TAMA300, except for the length of the arm cavities, and laser power and the mirror specifications. A large difference lies in spare mirrors with suspensions for cleaning the main cryogenic mirrors at room temperature. The sapphire mirror will have large optical loss and a fluctuation of birefringence. From a preliminary measurement of the optical loss, that amounted to $100 \mathrm{ppm} / \mathrm{cm}$ inside, we expect that this is not a major problem, considering the high thermal conductivity at the cryogenic temeperature against the thermal lensing effect. The estimated reduction of finnese by this loss does not degrade the recycling gain from the LCGT parameters, which will be reported soon.

\section{$6 \quad \mathrm{R} \& \mathrm{D}$ of LCGT}

For the realization of LCGT, we have been conducting developments of a cryogenic mirror of sapphire, the technique of high-reflective optical coating, and the conceptual design of a high-power laser.

\subsection{Cryogenic mirror}

The sensitivity of the middle frequency $(100-300 \mathrm{~Hz})$ is limited by thermalvibration noise, which is reduced by both decreasing the temperature and decreasing the internal mechanical loss, which increases the mechanical $\mathrm{Q}$ of the vibration. The source of thermal noise comes from both mirror internal vibration and swing noise of the pendulum supporting the mirror. The reduction of thermal noise is attained by cooling down the temperature of both the mirror, itself, and the suspension system.

If there were no heat production in the mirror, it would be an easy task to put all at the cryogenic temperature. However, high-power laser light produces a fractional loss of power at reflection and penetration of light in the mirror. Since we need a higher power to obtain a higher sensitivity, more heat is possibly produced in the higher sensitive detector. Since mirrors are suspended in a vacuum, there is no way to cool other than heat conductivity through suspended wires. Moreover, heat radiation at cryogenic temperature is negligible. As for the final way, we have found a sapphire crystal fiber for the candidate of the suspension wire, which has a possibility of high heat conduction at the cryogenic 
temperature. We have decided to adopt sapphire for the mirror material. The success of cryogenic mirror depends on how low the system is cooled without reducing the $\mathrm{Q}$ of the system.

In 1997, we checked the thermal distribution of the mirror with a heat source of an electric heater at the center of the mirror at the cryogenic temperature. The sapphire mirror was made by a single block of sapphire crystal, $10 \mathrm{~cm}$ in diameter and $6 \mathrm{~cm}$ in length. The mirror was put on a teflon block and fixed by two wounded sapphire fibers in half turn, the diameter of which is $250 \mu \mathrm{m}$ (in Fig. 11).

This was put into a vacuum inside a liquid-helium dewar (Fig. 12). Although the mirror should be suspended in a practical system, we put it upside down in fear of breaking the fiber when the vacuum chamber is inerted into the dewar. Figure 13 shows the measured distribution of the temperature, where the estimated power production on the mirror could be removed by these sapphire fibers. By adopting the designed parameters of TAMA, we could estimate the lowest temperature of the mirror as being less than $20 \mathrm{~K}$. This means a two-order improvement in the sensitivity, assuming no degradation of the mechanical $Q$ [8].

In 1998, we measured the mechanical Q of both the sapphire rod and the sapphire fiber, itself. This time, the sapphire rod was suspended as in Fig. 14 with an appropriate exciter and vibration transducer.

Since the most important vibration mode is the lowest axial one, we measured this mode and the next lowest one, as shown in Fig. 15, the mode patterns of which were numerically calculated by a finite-element method.

Figure 16 shows the dependence of $\mathrm{Q}$ on the temperature. $\mathrm{Q}$ at temperature lower than liquid nitrogen is the intrinsic $\mathrm{Q}$ of the material, because it is hard to find the origin of the loss. A detailed discussion is given in a paper [9].

We finish the description of $\mathrm{Q}$ by mentioning a measurement of the fiber of suspension. We do not argue about the loss mechanism of the fiber, but measure for a practical suspension condition. The measured value has confirmed the high quality of sapphire fiber in the cryogenic temperature. The best $\mathrm{Q}$ reached $1 \times 10^{7}$, which enables the $\mathrm{Q}$ of the suspension to be typically more than $1 \times 10^{9}$. This result will be published soon.

We are developing a system to observe a direct reduction of the thermal vibration by cooling the system.

\subsection{High reflectance optical coating}

A high-quality coating had been accomplished by ion-beam sputtering for smallsized mirrors before. TAMA and Japan Aviation Electronics Corporation have developed a high-quality mirror in large size, such as the TAMA main mirror. The measured optical loss was less than $50 \mathrm{ppm}$, which is the target value [10]. For smaller size the loss is better than $2 \mathrm{ppm}$ and this quality was applied to the $20 \mathrm{~m}$ prototype Fabry-Perot Interferometer to test power recycling. The maximum gain of recycling was 12 , which is the best at present for a suspended mirror system in the world. 


\subsection{Optical quality measurement}

Recently, we developed a measurement of the optical loss at cryogenic temperature. Since the heat capacity of sapphire is small and the heat conductivity is large at the cryogenic temperature, the heat-up temperature is a good measure of the optical loss inside the mirror. If we measure the scattering loss at the same time, we can precisely estimate its optical loss apart from its scattering loss. Practically, since it is hard to measure scattering inside the cryostat, this is done by another experimental setup working at room temeperature. We have already made a preliminary measurement of both the heat-up one and the scattering one. It is too early to give any numbers.

\subsection{Conceptual design of high-power laser}

To realize an $100 \mathrm{~W}$ laser, we adopt a power amplifier to a $10 \mathrm{~W}$ laser developed for TAMA 300 interferometer. The amplifier is similar to MOPA (Master Oscillator, Power Amplifier), which was developed by Lightwave Electronics Corporation. Because the module was reported to amplify not only laser power, but also any intensity noise, we must reduce it by transmission filtering of a FabryPerot cavity, called Pre-Mode-Cleaner. The cavity, which consists of ultra-low loss mirrors and a ULE spacer, is a rigid-ring optical cavity with a finesse of about 1000. Figure 17 shows the conceptual design of the high-power laser.

\section{Cost estimation of LCGT}

The cost of the construction determines the total cost. Since the detector is built in an underground tunnel at Kamioka mine, the cost of installation and that of maintenance during construction are not negligible. The main expenditures as as follows: of

- Civil engineering of the tunnel

- Machining and installing Vacuum ducts and chambers

- Interferometer system

- Cooling devices for cryogenic mirrors

- Data-taking system

- Maintenance cost of the facility during construction

\section{Schedule and Organization of LCGT}

The most important strategy of LCGT is to be on time for catching the first event in the world competition. Therefore, completion of the construction should be achieved before 2007, when the improvement of the initial LIGO will be finished. LIGO plans to begin observations by the initial system in 2002, and plans to apply several improvements for four years after 2002. We suppose that the target year of the second observation is 2007. This may depend on 
a possible delay in the construction of the initial system. However, we do not expect any delay of LIGO. Since LCGT is a five-year project, it must start in 2002 at the latest.

The organization of LCGT succeeds to TAMA. Although many young scientists have grown up during the development of TAMA, we still need to hire more to begin LCGT. The staff of ICRR will have responsibility for the construction and organization of scientific research groups as users of LCGT.

\section{References}

[1] A. Abramovici, W.E. Althouse, R.W.P. Drever, Y. Gursel, S.Kawamura, F.J. Raab, D. Shoemaker, L. Sievers, R.E. Spero, K.S. Thorne, R.E. Vogt, R. Weiss, S.E. Whitcomb, M.E. Zucker, Science 256 (1992) 325: A. Abramovicu, W. Althouse, J. Camp, D. Durance, J.A. Giaime, A. Gillespie, S. Kawamura, A.Kuhnert, T. Lyons, F.J. Raab, R.L. Savage Jr, D. Shoemaker, L.Sievers, R. Spero, R. Vogt, R. Weiss, S. Whitcomb, M. Zucker, Improved sensitivity in a gravitational wave interferometer and implications for LIGO, Phys. Lett. A 218 (1996) 157.

[2] The VIRGO Collaboration, VIRGO Final Conceptual Design, 1992.

[3] K. Danzmann, H. Luck, A. Rudiger, R. Schilling, M. Schrempel, W. Winkler, J. Hough, G.P. Newton, N.A. Robertson, H. Ward, A.M. Campbell, J.E. Logan, D.I. Robertson, K.A. Strain, J.R.J. Benner, V. Kose, M. Kuhne, B.F. Schutz, D. Nicholson, J. Shuttleworth, H. Welling, P. Aufmuth, R. Rinkleff, A. Tunnermann, B. Willke, Proposal for a $600 \mathrm{~m}$ Laser-Interferometric Gravitational Wave Antenna, Max-Planck-Institut fur Quantenoptik Report 190, Garching, Germany, 1994.

[4] K. Tsubono, $300 \mathrm{~m}$ laser interferometric gravitational wave detector (TAMA300) in Japan: Proc. First E. Amaldi Conference on Gravitational Wave Experiemnts, June, 1994, World Scientific, Singapore, 1995: K. Kuroda, Y. Kozai, M-K Fujimoto, M. Ohashi, R. Takahashi, T. Yamazaki, M.A. Barton, N. Kanda, Y, Sato, N. Kamikubota, Y. Ogawa, T. Suzuki, N. Kawashima, E. Mizuno, K. Tsubono, K. Kawabe, N. Mio, S. Moriwaki, A. Araya, K. Ueda, K. Nakagawa, T. Nakamura and Members of TAMA group, Status of TAMA, Proc. Int. Conf. on Gravitational Waves; Sources and Detectors, edit. I. Ciufolini and F. Fidecaro, World Scientific, 1997.

[5] E.S. Phinney, The rate of nuetron star binary merges in the universe: minimal predictions for gravity wave detectors, Astrophysical J. 380 (1991) L17.

[6] J.H. Taylor and J.M. Weisberg, Further experimental tests of relativistic gravity using the binary pulsar PSR 1913+16, Astrophysical J., 345 (1989) 434.

[7] C.M. Will, Theory and experiment in gravitational physics, Chap. 10, Cambridge University Press, 1993 
[8] T. Uchiyama, D. Tatsumi, T. Tomaru, M.E. Tobar, K. Kuroda, T. Suzuki, N. Sato, A. Yamamoto, T. Haruyama, T. Shintomi, Cryogenic cooling of a sapphire mirror-suspension for interferometric gravitational wave detectors, Phys. Lett. A 242(1998)211.

[9] T. Uchiyama, T. Tomaru, M.E. Tobar, D. Tatsumi, S. Miyoki, M. Ohashi, K. Kuroda, T. Suzuki, N. Sato, T. Haruyama, A. Yamamoto, T. Shintomi, Mechanical quality factor of a cryogenic sapphire test mass for laser interferometric gravitational wave detectors, Phys. Lett. A, submitted.

[10] S. Sato, S. Miyoki, M. Ohashi, M-K Fujimoto, T. Yamazaki, M. Fukushima, A. Ueda, K. Ueda, K. Watanabe, K. Nakamura, K. Etoh, N. Kitajima, K. Ito and I. Kataoka, Loss factors of mirrors for a gravitational wave antenna, Appl. Opt. 38 (1999) 2880.

[11] Y. Saito, N. Matsuda, Y. Ogawa, and G. Horikoshi, Vacuum system design for the $300 \mathrm{~m}$ gravitational wave laser interferometer in Japan (TAMA300), Vacuum 47 (1996) 609:Y.Saito, Y.Ogawa, G.Horikoshi, N.Matuda, R.Takahashi, M.Fukushima, Vacuum system of the $300 \mathrm{~m}$ gravitational wave laser interferometer in Japan (TAMA300), Vacuum 53 (1999) 353.

[12] Internal report in Japanese (1997).

[13] S. Telada and TAMA collaboration, Doctor Thesis (1999).

[14] A. Araya, S. Telada, K. Tochikubo, S. Taniguchi, R. Takahashi, K. Kawabe, D. Tastumi, T. Yamazaki, S. Kawamura, S. Miyoki, S. Moriwaki, M. Musha, S. Nagano, M-K. Fujimoto, K. Horikoshi, N. Mio, Y. Naito, A. Takamori and K. Yamamoto, Absolute-length determination of a longbaseline Fabry-Perot cavity by means of resonating modulation sidebands, Appl. Optics 38 (1999) 2848.

[15] K. Tchikubo and TAMA collaboration (Doctor Thesis, submitted)

[16] S. Miyoki, T. Uchiyama, T. Tomaru, D. Tatsumi, H. Ishiduka, M. Ohashi, K. Kuroda, A. Ueda, T. Suzuki, N. Sato, T. Haruyama, A. Yamamoto, T. Shintomi, Cryogenic contamination of an ultra-low loss mirror for cryogenic laser interferometric gravitational wave detector, in preparing. 


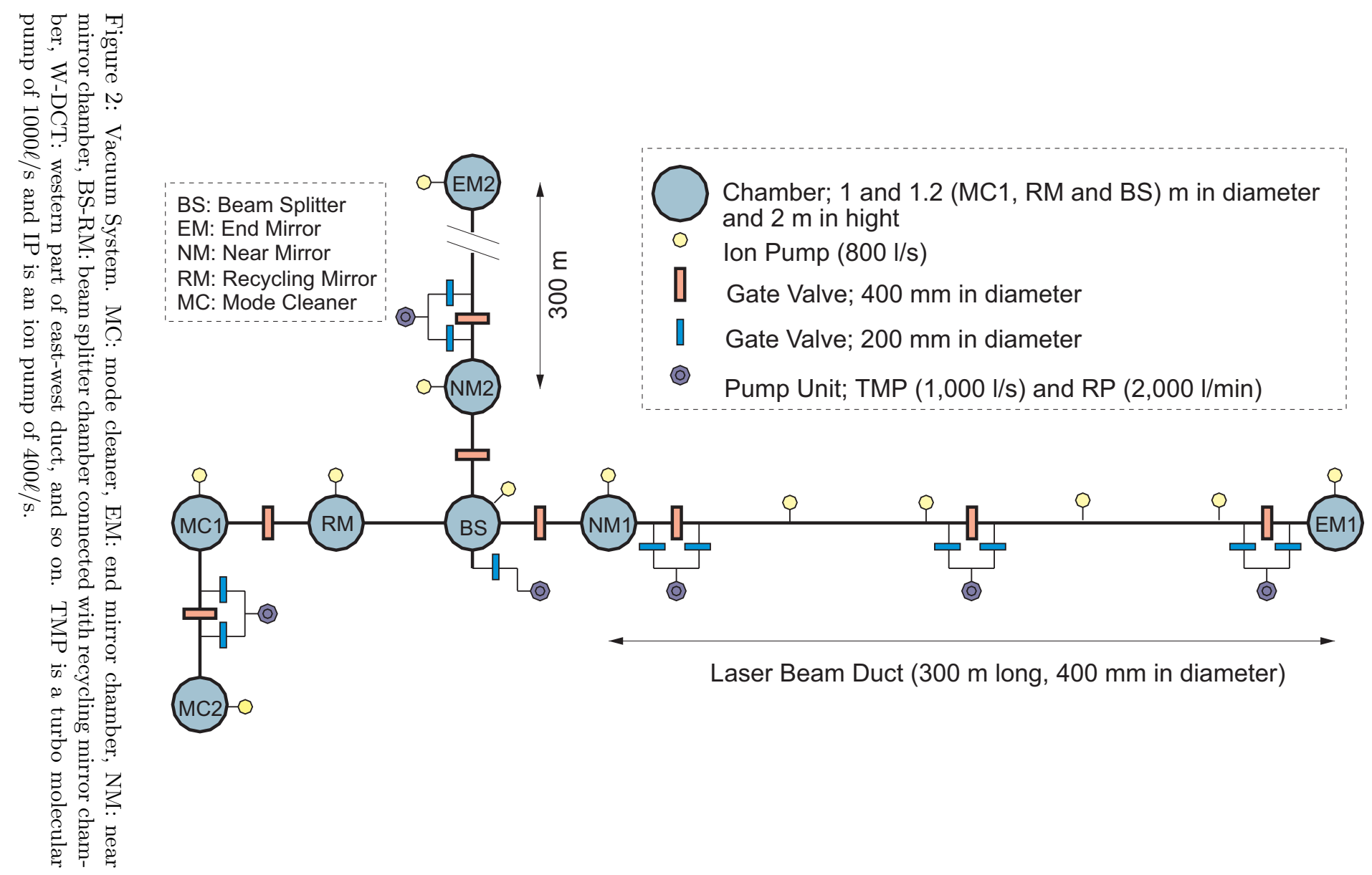




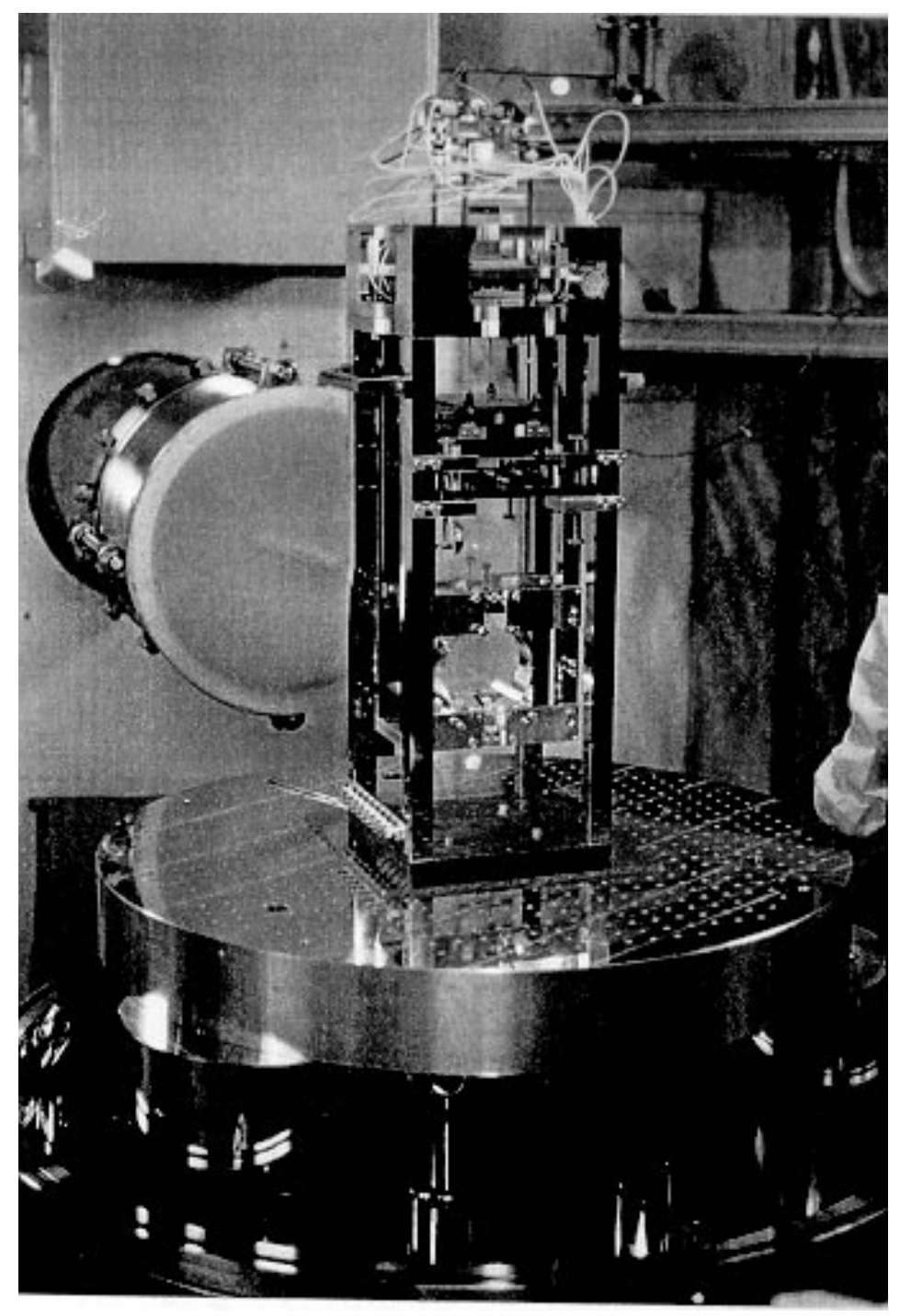

Figure 3: Main mirror suspended by a suspension placed on an optical breadboard supported by three stacks inside a vacuum chamber with the upper part open. 


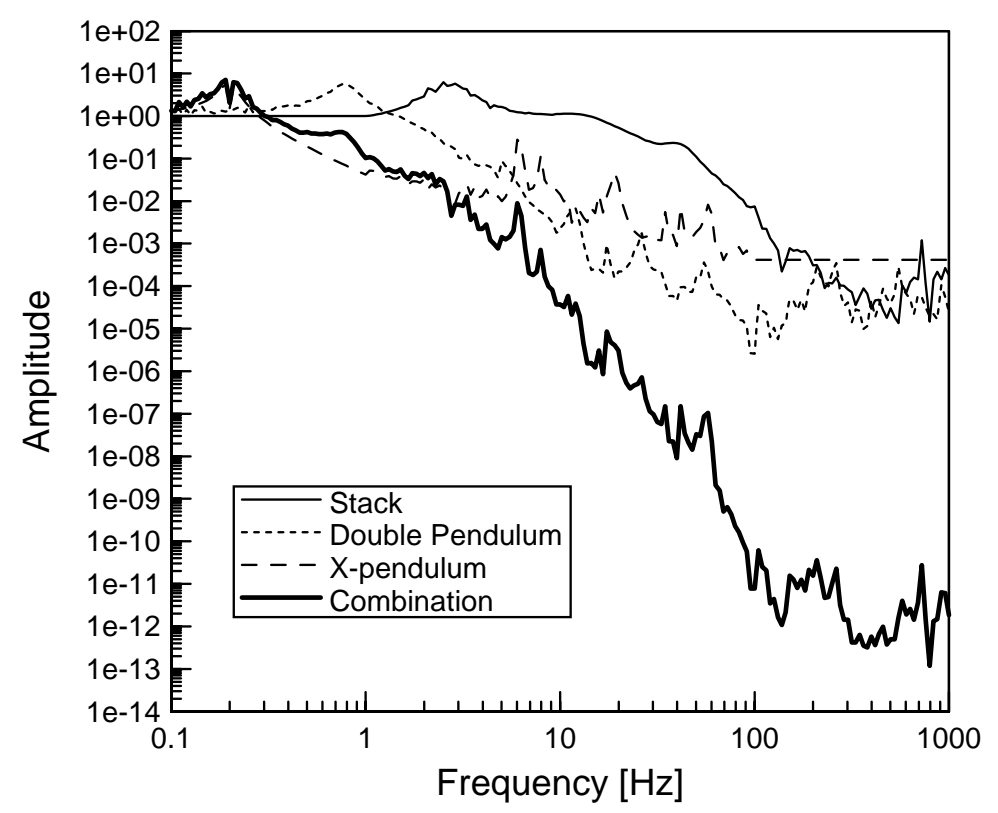

Figure 4: Transfer function of the isolation system. Every part (the stack, the double suspension and X-pendulum) was measured independently and composed later. The measurement was performed in the horizontal direction along the laser beam optical axis.

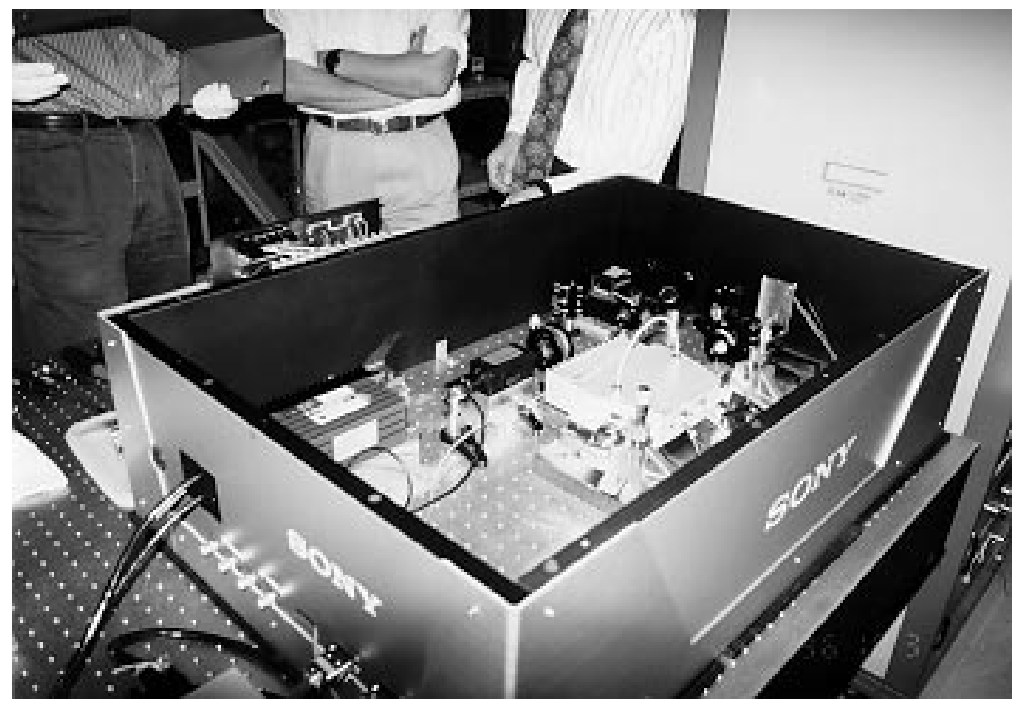

Figure 5: $10 \mathrm{~W}$ injection locked Nd:YAG laser system developed by Sony Corporation. 
S.osuəs uo!̣!̣sod urəg : $\varepsilon^{\prime} \tau \mathcal{E}^{\prime} I \mathcal{E}^{\prime} 0 \mathcal{E}$

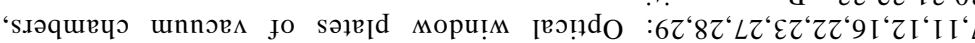

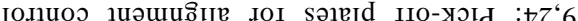

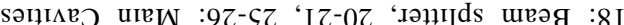

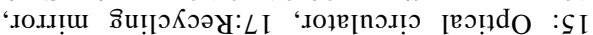

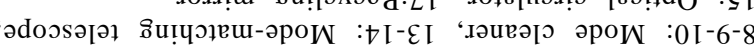

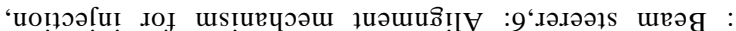

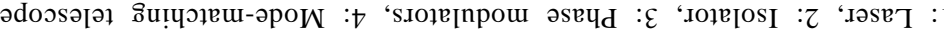

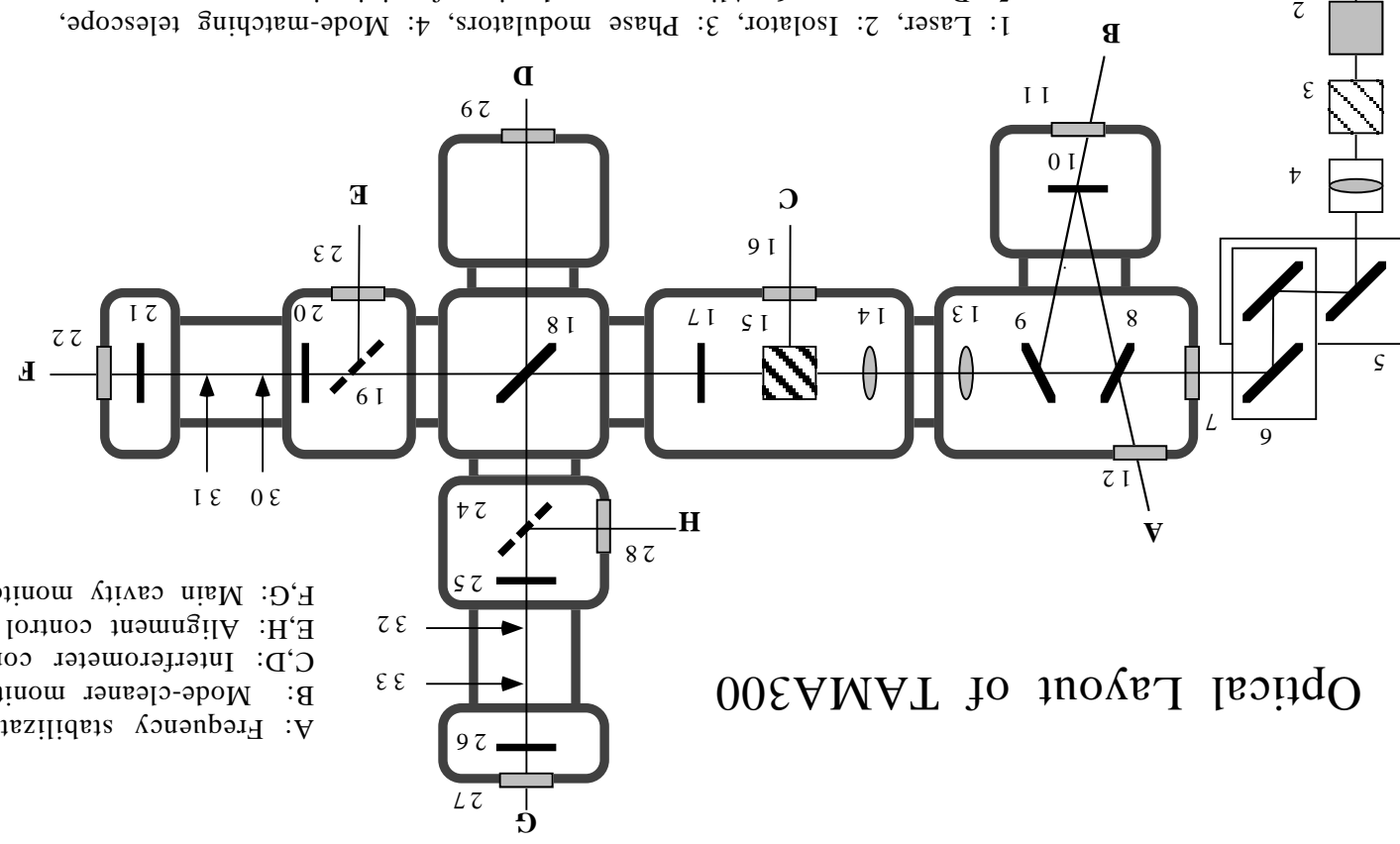




\section{Displacement noise level of TAMA300}

(May, 29, 1999)

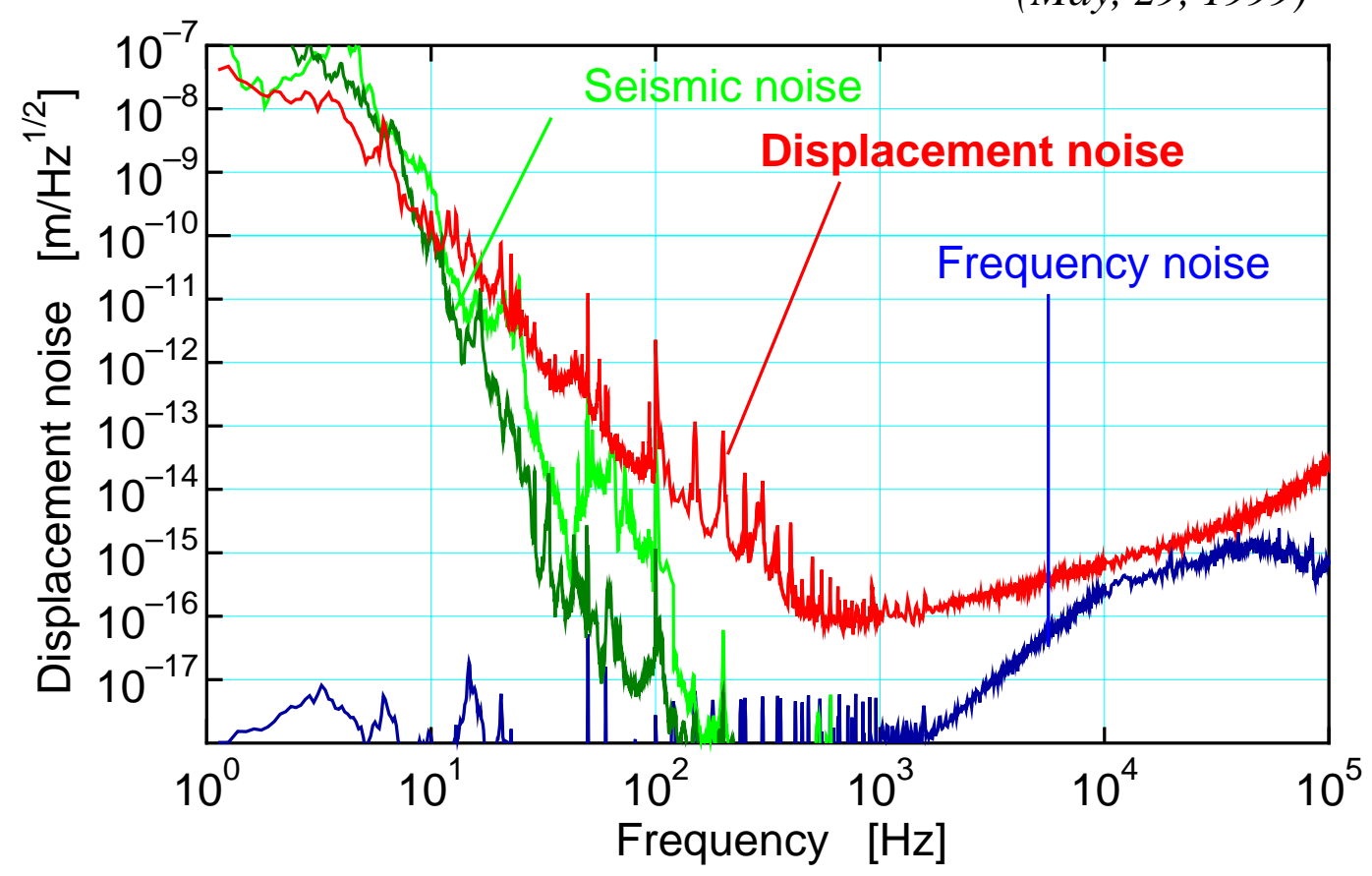

Figure 7: Sensitivity curve in the $300 \mathrm{~m} \mathrm{FP}$ interferometer. The optical configuration is the same as the final one, except for recycling. 


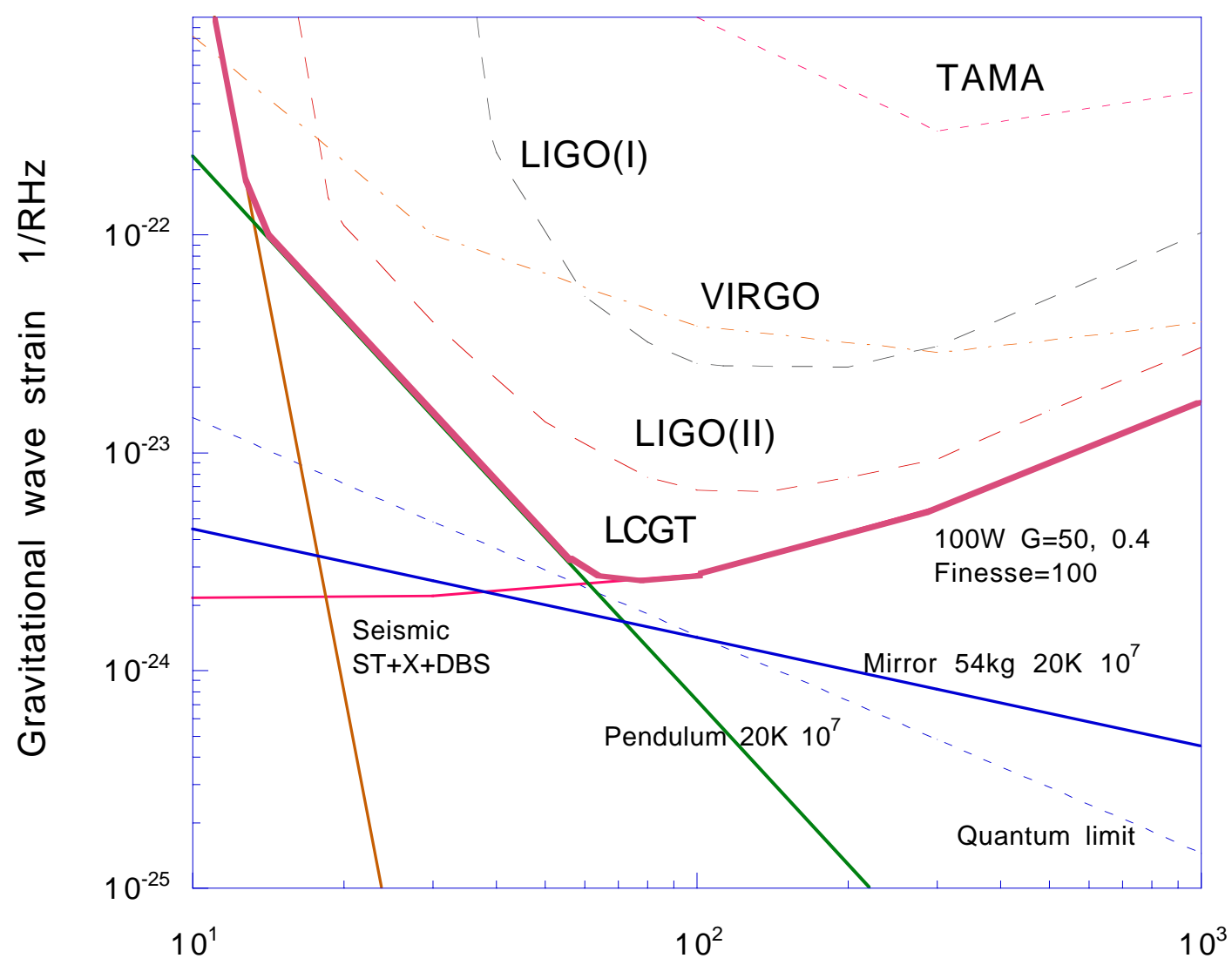

Frequency, $\mathrm{Hz}$

Figure 8: Senstivity of LCGT. The specifications are listed in Table 2. 


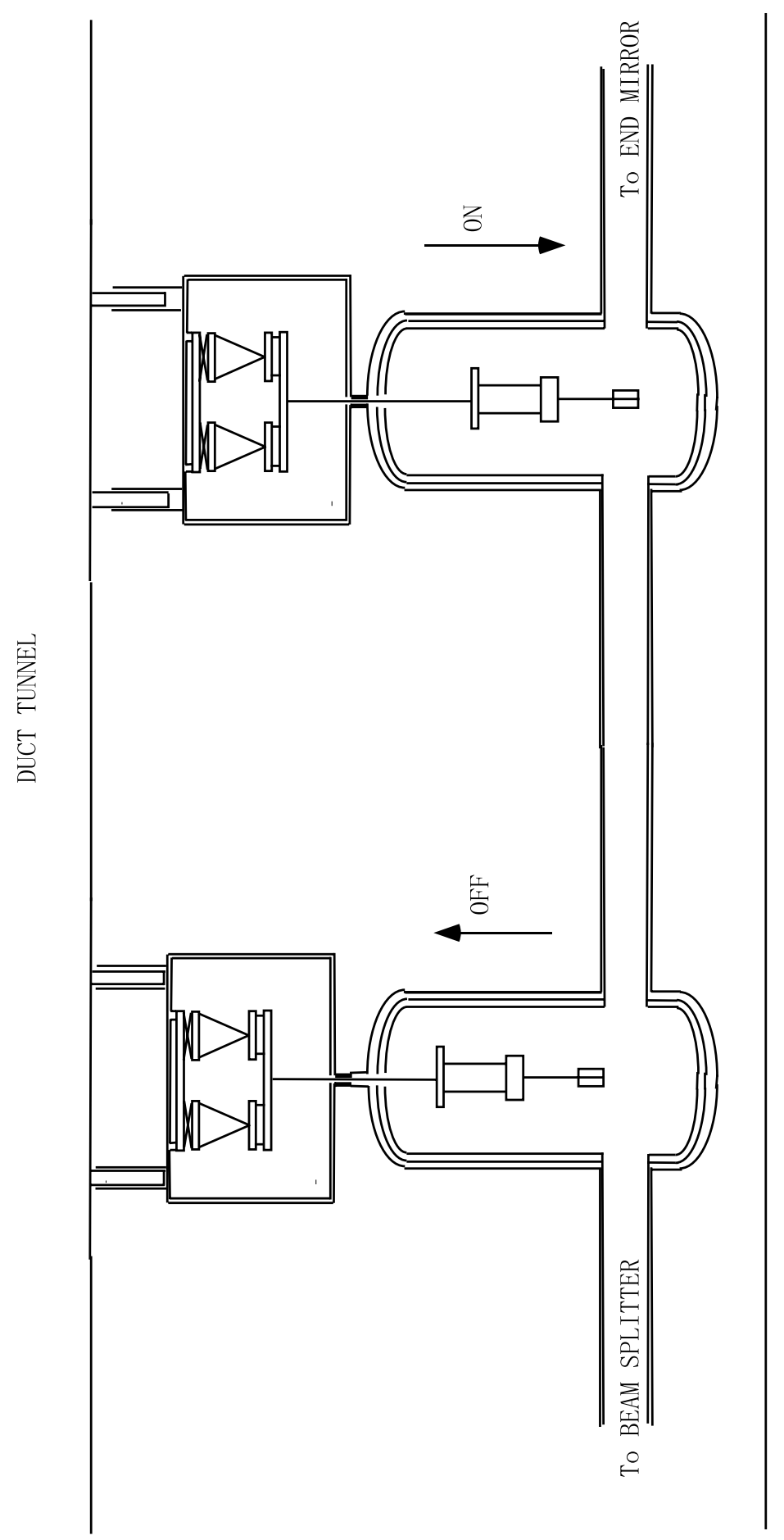

Figure 9: Vacuum system of LCGT is similar to that of TAMA, except for the above mirror switching system for contamination purging. 


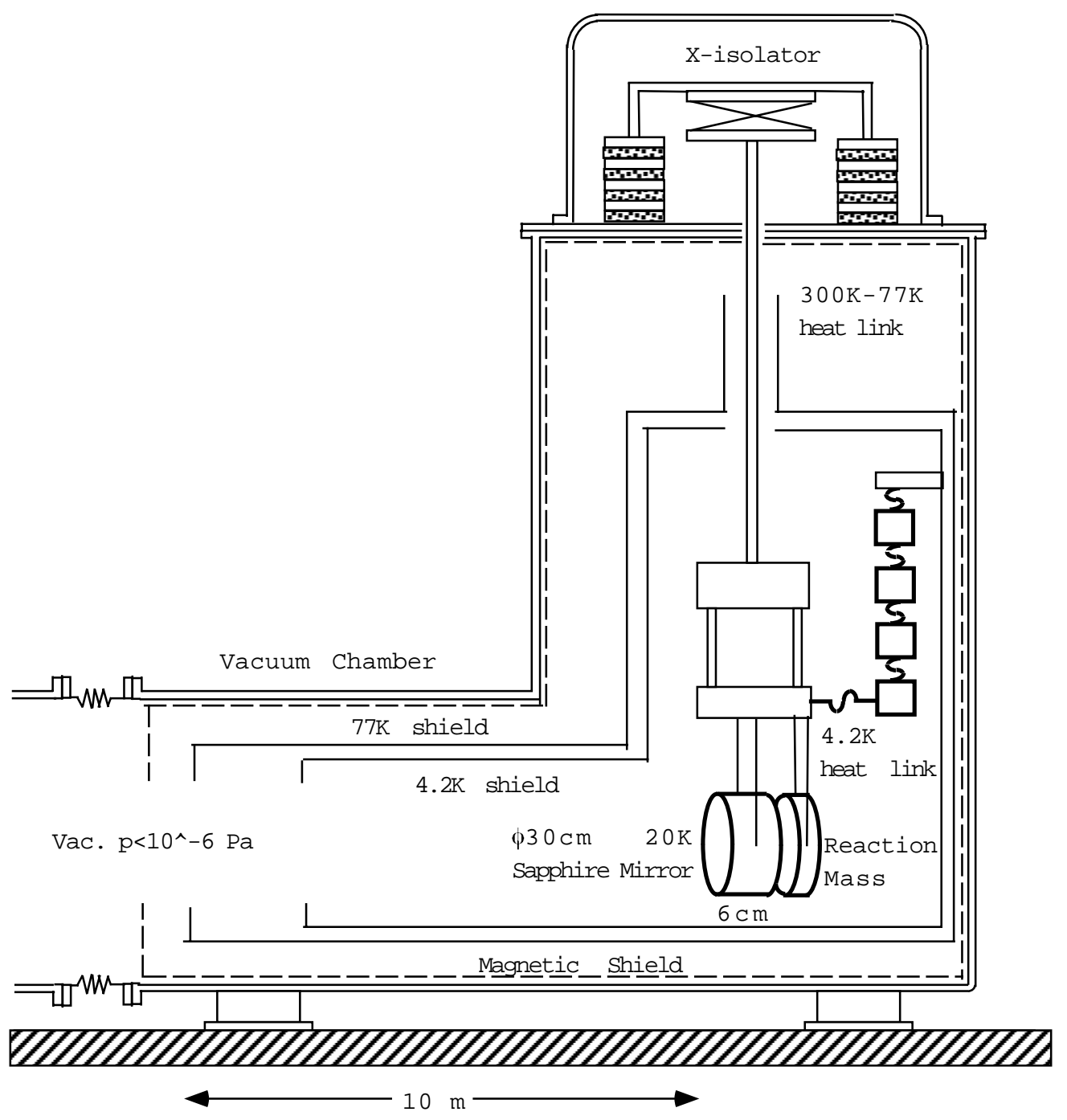

Figure 10: Overview of the cryogenic suspension system. The mirror is suspended by sapphire fibers connected to a platform that has a heat link to the helium liquid tank inside the vacuum. The platform is suspended with a G-10 rod connected through the center hole of the helium tank to an isolation table suspended by a double X-isolator. 


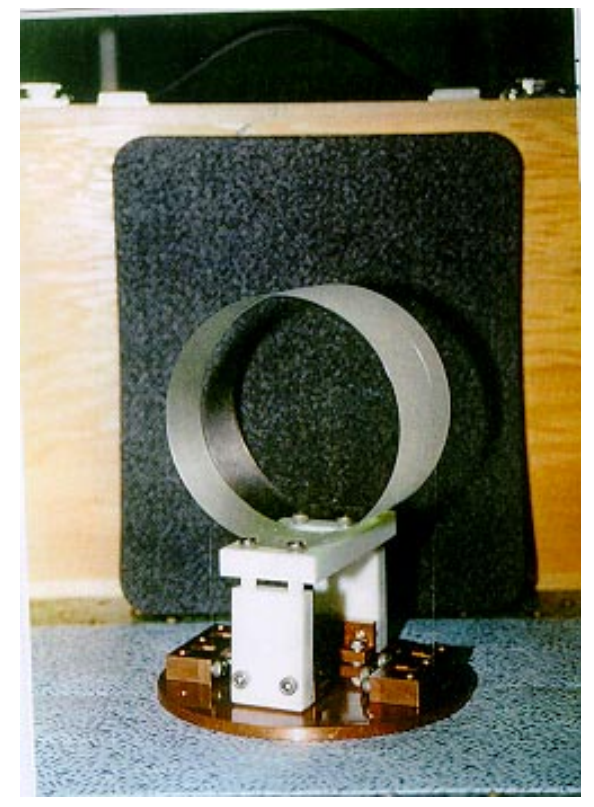

Figure 11: Sapphire mirror on a teflon block and fixed by two wounded sapphire fibers in half turn, the diameter of which is $250 \mu \mathrm{m}$

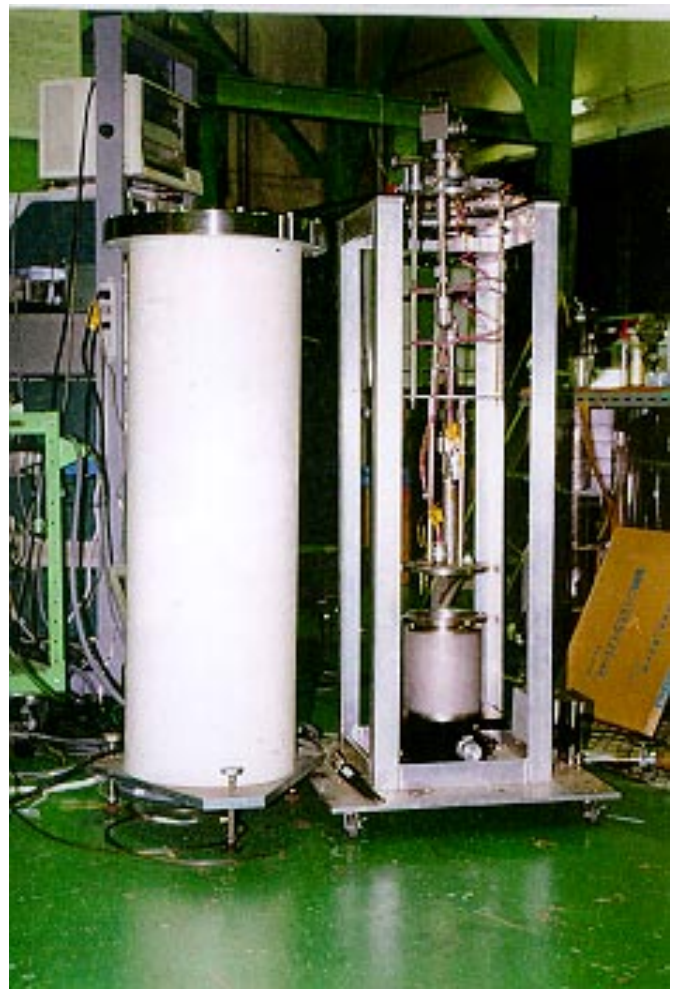

Figure 12: Sapphire mirror placed in a vacuum insert in a liquid-helium dewar. 


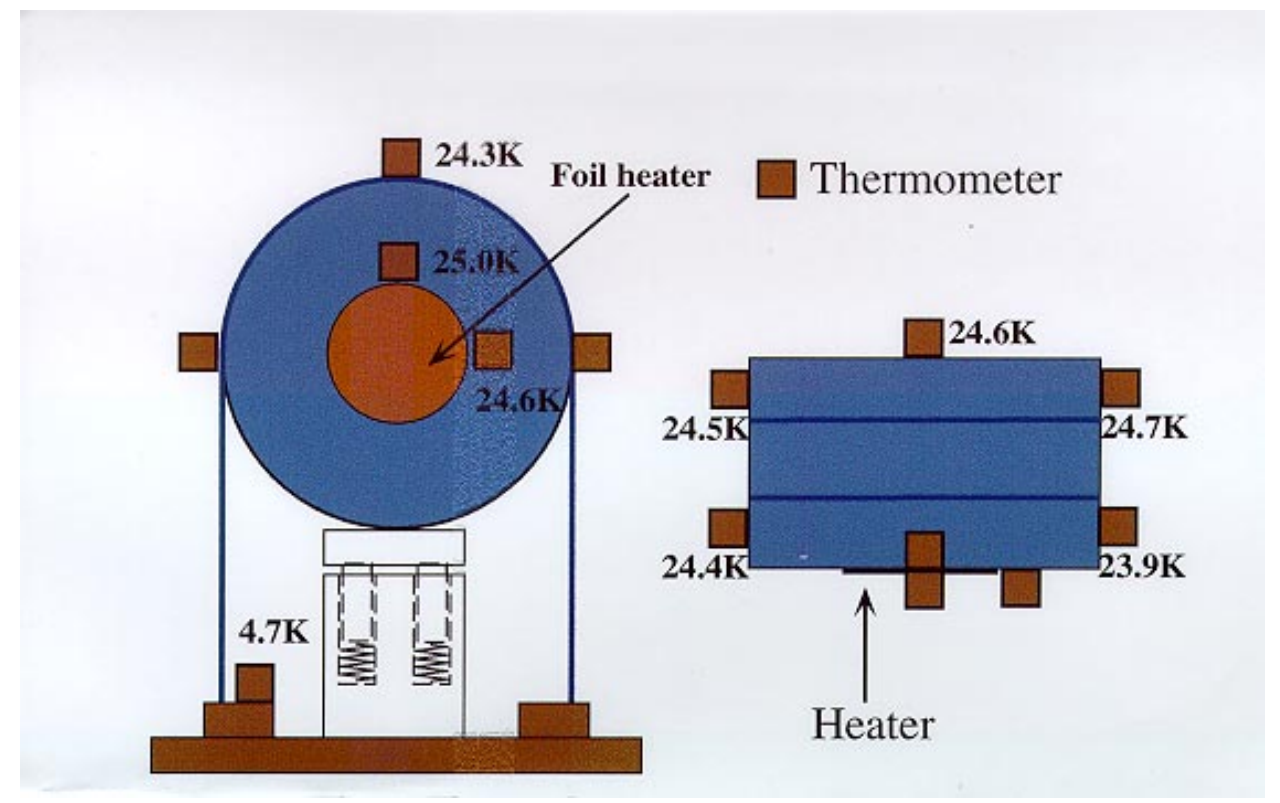

Figure 13: Measured distribution of the temperature showing that the estimated power production on the mirror could be removed by these sapphire fibers.

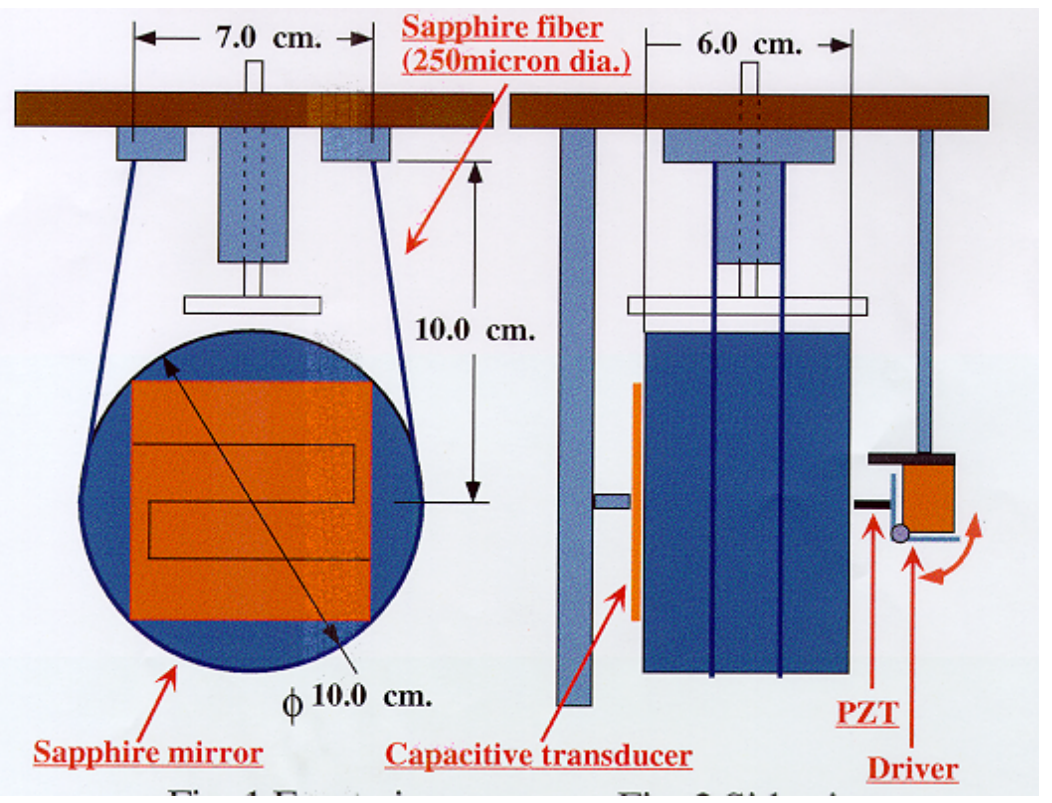

Figure 14: $10 \mathrm{~cm}$ sapphire rod suspended by two sapphire fibers, $250 \mu \mathrm{m}$ in diameter. The capacitive transducer sensed the vibration with negligible mechanical loss and vibrational modes were excited by the PZT driver attached on a removable lever. 


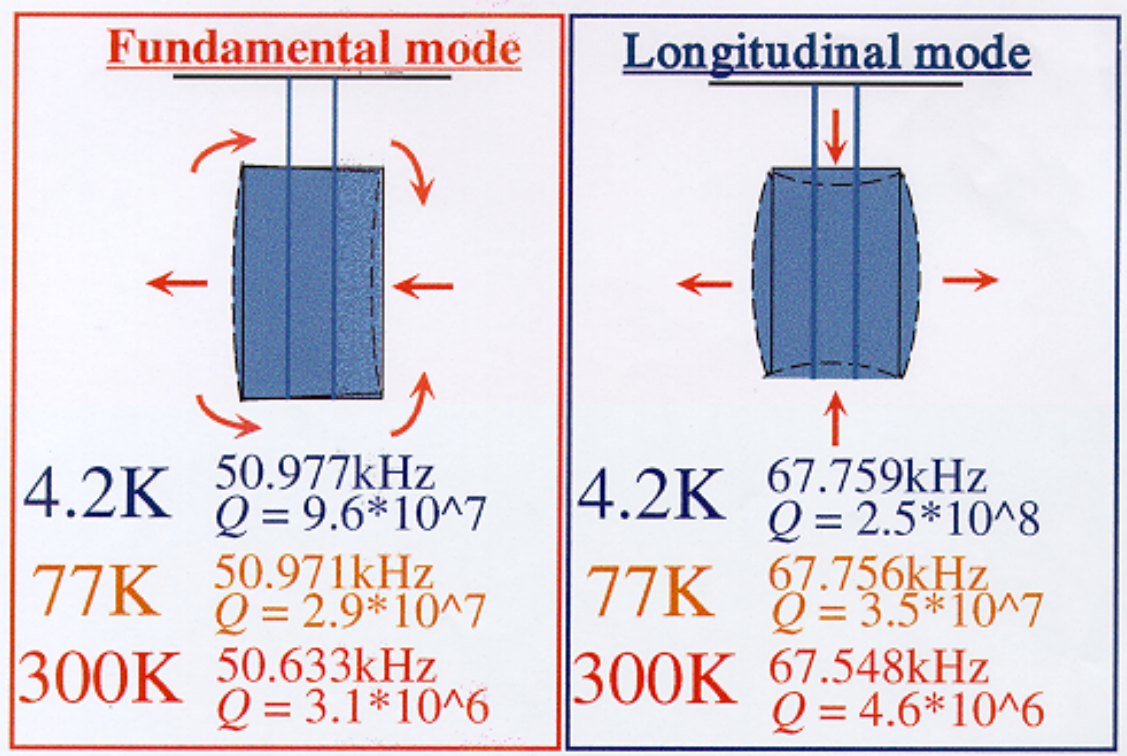

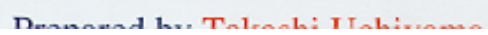

Figure 15: Q of internal vibration modes with axial symmetry. Mode patterns were numerically calculated by a finite element method.

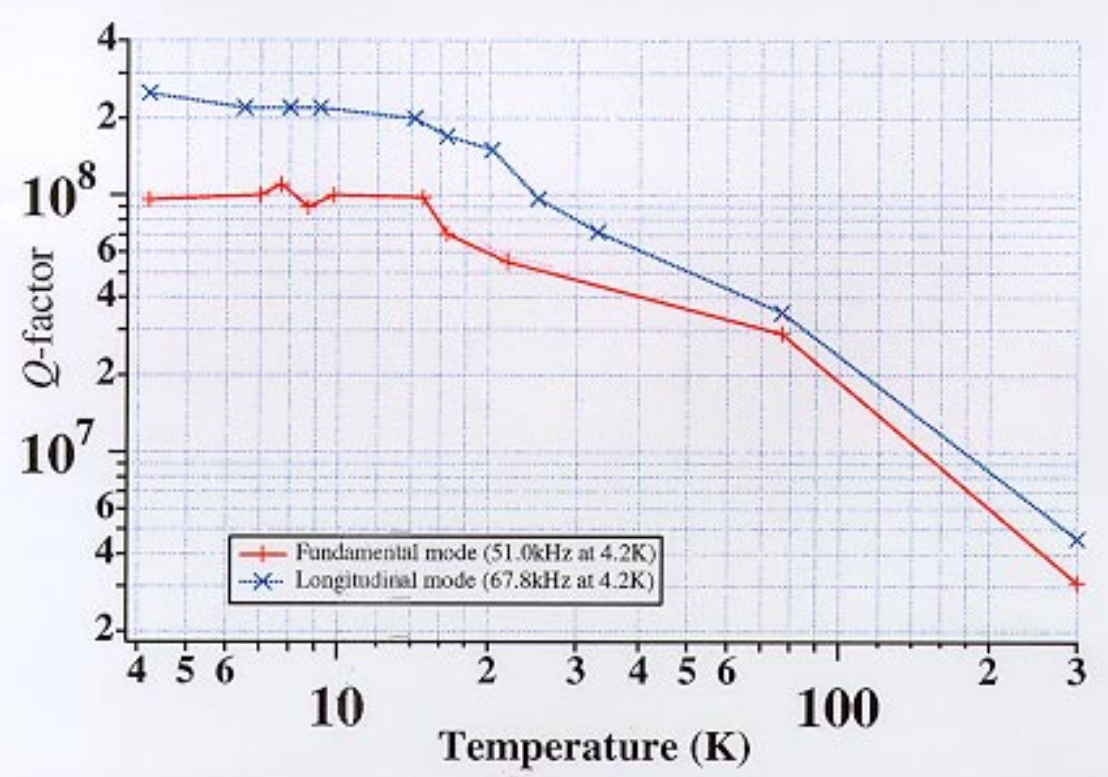

Figure 16: $\mathrm{Q}$ of internal vibration modes in cryogenic temperature. The $\mathrm{Q}$ in a practical suspension was first measured in this experiment. 


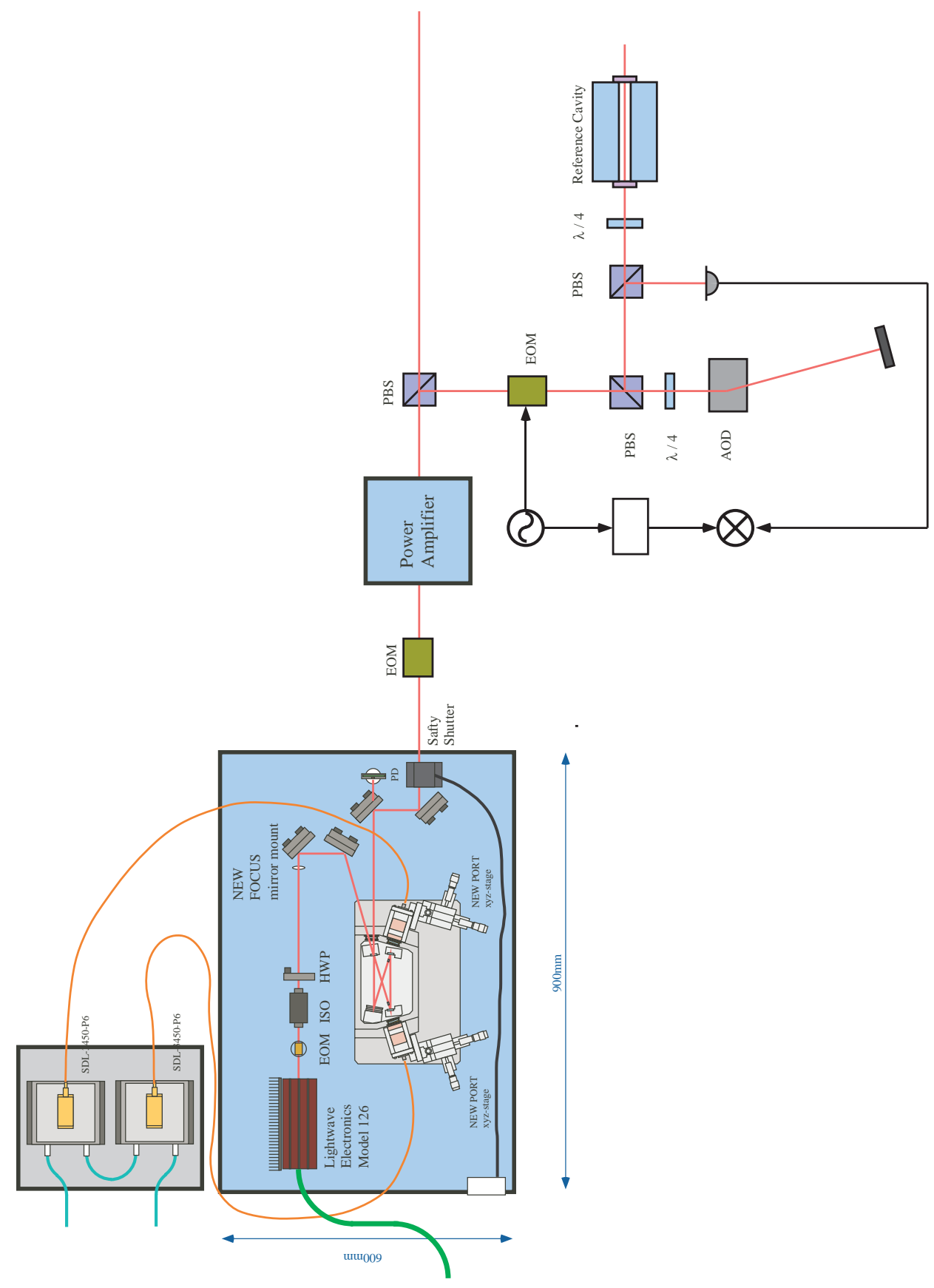

Figure 17: High-power laser source with high-frequency stability considered to be built by applying an optical amplifier to the output of a master oscillator, such as an injection locking laser system like that as used in TAMA. 